\section{Genetical bouillabaisse}

\author{
Aquatic Genomics: Steps Toward a Great \\ Future \\ N Shiizu, T Aoki, I Hirono and F Takashima (eds) \\ Springer-Verlag, Tokyo; 2003. 432 pp. \\ Price €139.95, hardback. ISBN 4-431-70331-4
}

Heredity (2003) 91, 545. doi:10.1038/sj.hdy.6800362

\section{Reviewed by JA Beardmore}

Work on many aspects of the genetics of aquatic organisms has made considerable progress in the last couple of decades helped, no doubt, by the dramatic growth rate of the aquaculture industry worldwide as well as by many technological advances in molecular genetics. It could, thus, reasonably be judged to be an apposite moment for a book on the genomics of aquatic species to appear. However, neither from the preface nor the contents pages of this volume was I able to infer the precise purpose of the book, nor indeed, what genomics comprises. I therefore asked three experts on the genetics of aquatic species to define genomics and received three significantly different answers. This unexpected taxonomic diversity of understanding, as to what exactly genomics constitutes, is doubtless reflected more widely and possibly accounts for some opacity of intent in the ordering of the contents of this book.

The editors relate that the book follows the holding of the First Aquatic Genomics Symposium in 2000 in Tokyo and '... was designed as a collection of advanced knowledge in aquatic genomics'. It is certainly a collection, and one with many absorbing and stimulating contributions to advanced knowledge, but it is difficult to discern any clear rationale that determines the specific objectives of the volume. It is, thus, particularly unfortunate that the editors have not taken the step of producing a synthesis that attempts to associate specific targets on which they have tried to focus with contributions in the separate chapters.

The number of recognised fish species is close to 25,000 - more than half of all known vertebrates. They display great diversity in many respects, partly at least because they do not represent a single phyletic lineage but rather five lineages that are separated at a very early stage. It is, then, not surprising that in almost all of the chapters fish are the organisms under investigation with a nod to crustaceans and a couple of contributions on oysters. Plants, mammals, reptiles, amphibians and insects of aquatic habitats are not represented but there is probably little work on genomics of these groups being carried out.

The quality and quantity of the 38 individual chapters varies. A few, relatively short, contributions present accounts of work performed with little or no discussion of significance of the results obtained. Most, however, of them are more informative and it is particularly helpful that there are many contributions from Japan and China from individuals and groups, whose work, in some instances, may otherwise not always be well known and accessible to Western colleagues.

Much, but not all, of the work reported in the earlier chapters could be said to be concerned with structural genomics as it deals with mapping and the actual and potential applications of map data. These include adaptive radiation of species, genetic improvement of cultured strains, genome expansion and comparative genomics. It is interesting to learn that for fish fairly complete information is available on the genomes of three species. I also particularly appreciated a discussion of the way in which genomic approaches have been used to produce better understanding of heterosis and improve yield in Pacific oysters.

The middle chapters cover a wide variety of topics, which broadly deal with functional genomics ranging, for example, from the cloning of sex determining sequences in medaka through expression of carp myosin mRNAs during temperature acclimatisation to genome fluidity influencing melanoma formation and sex determination in Xiphophorus. The last third or so embraces a diversity of topics in which for example the use of transgenics in the study of gene regulation and in aquaculture, use of DNA markers in marker assisted selection, and applications of markers to broodstock management in aquaculture are discussed.

An index of key words is provided. This is, however, sparse and incomplete. For example, 'transgenic fish' rates only one page reference, despite the fact that at least eight chapters discuss transgenic fish in some detail, likewise genomics is (usefully) defined on p. 36 but this does not secure a mention in the index while the QTL entry refers only to one chapter despite discussion of these in several other chapters. There is no author index nor an organism index. A separate bibliography is provided for each chapter. These are generally comprehensive and most are up to date but the inadequate level of indexing makes bibliographical navigation for the reader more difficult than it should be.

It is a pity that the effective exploitation of this volume is handicapped by the structural deficiencies mentioned above, which are even less acceptable bearing in mind the substantial cost of the volume. Despite the critical comments, overall this book does provide a wealth of technical data and interesting theoretical discussion from a body of front line experts and it has valuable messages for geneticists, evolutionary biologists, aquaculturists, fish biologists, immunologists and students of fish disease. It should also provide a useful platform for planning and executing work needed to achieve the 'great future' envisaged in the subtitle.

JA Beardmore

Margam Building, School of Biological Sciences, University of Wales Swansea, Singleton Park, SWANSEA SA2 8PP, UK 